\title{
Tumores cardíacos de resorte quirúrgico. Serie de Casos
}

\section{Cardiac tumors requiring surgical resection. Number of cases}

\author{
Nancy Garay', Soledad Alvarez', Marcos Melgarejo', Carlos Verón', Maria Esther Meza', \\ José Bellasai ${ }^{1}$
}

\section{RESUMEN}

Introducción: Los tumores cardiacos son infrecuentes en la edad pediátrica, en su mayoría son primarios y benignos (90\%), siendo los más frecuentes los Rabdomiomas, Fibromas y Mixomas. Las indicaciones quirúrgicas están relacionadas al efecto hemodinámico o mecánico, arritmias y/o disfunción ventricular que ocasionan, más que a su origen anatomopatológico de benignidad o tamaño tumoral. En los tumores malignos o metastásicos la conducta a seguir la determina el origen primario del tumor, así como el estadio en que se encuentra cada paciente. Objetivos: Presentar la casuística y conducta médica de pacientes con Tumor Cardiaco sometidos a exéresis quirúrgica, así como su evolución inmediata y a mediano plazo. Material y Métodos: Análisis retrospectivo, observacional, mediante la revisión de datos de historias clínicas de niños diagnosticados con tumores cardiacos por ecocardiografía transtorácica. Resultados: En nuestra casuística, 4 de los 5 tumores fueron benignos ( $80 \%$ ) y 1 maligno (20\%); hubo $100 \%$ de coincidencia del hallazgo por Ecocardiografía bidimensional transtorácico de imagen de masa tumoral con el hallazgo quirúrgico; el diagnóstico histopatológico informó Mixoma en 3 casos (60\%), 1 paciente $(20 \%)$ Rabdomioma gigante y 1 paciente (20\%) Carcionoma Mucoepidermoide de Timo. Encontramos correlación positiva entre el diagnóstico clínico-imageneológico y los resultados histopatológicos. Discusión: Los tumores cardiacos primarios benignos, tuvieron indicación de exéresis quirúrgica basados en su localización y repercusión hemodinámica obstructiva a las vías de entrada ventriculares. En el caso del carcinoma mucoepidermoide de timo de bajo grado de malignidad, el diagnóstico y resección quirúrgica precoz permitió la exéresis total, no requiriendo terapia adicional. La evolución posoperatoria sin complicaciones a excepción de un caso (20\%) que requirió asistencia con ECMO. Todos

\section{ABSTRACT}

Introduction: Cardiac tumors are uncommon in the pediatric age group, most are primary and benign (90\%), the most frequent being rhabdomyomas, fibroids and myxomas. Indications for surgical treatment are related to the hemodynamic or mechanical effect, arrhythmias and / or ventricular dysfunction they cause, rather than their pathological origin, benignity or tumor size. In malignant or metastatic tumors, the treatment is determined by the primary origin of the tumor, as well as the patient's staging. Objectives: To present the types of tumors and management of patients with cardiac tumors who underwent surgical excision, as well as the short and medium-term outcomes. Material and Methods: This was a retrospective and observational study, performed by reviewing data from the medical records of children diagnosed with cardiac tumors by transthoracic echocardiography. Results: In our series, 4 of the 5 tumors were benign (80\%) and 1 malignant (20\%); there was $100 \%$ coincidence of the finding by two-dimensional transthoracic echocardiography of tumor mass image with the surgical findings; Histopathological diagnosis reported myxoma in 3 cases $(60 \%), 1$ patient $(20 \%)$ had a Giant rhabdomyoma and 1 patient (20\%) had a Thymus Mucoepidermoid Carcionoma. We found a positive correlation between clinical/imaging diagnosis and histopathological results. Discussion: The benign primary cardiac tumors in this series were referred for surgical excision based on their location and obstructive hemodynamic repercussions related to the ventricular entry pathways. In the case of the thymus mucoepidermoid carcinoma with a low degree of malignancy, the diagnosis and early surgical resection allowed total excision; it did not require additional therapy. Postoperative courses were without complications except for one case $(20 \%)$ that required assistance with ECMO. All were discharged and remain

\footnotetext{
${ }^{1}$ Departamento de Cardiología. Hospital General Pediátrico Niños de Acosta Ñú

Correspondencia: Nancy Garay Correo: nancygareche@hotmail.com

Conflicto de interés: Los autores declaran no poseer conflicto de interés

Recibido: 10/08/2019 Aceptado: 25/10/2019

Doi: https://doi.org/10.31698/ped.46032019007
}

(cc) BY Este es un artículo publicado en acceso abierto bajo una Licencia Creative Commons CC-BY 4.0 
fueron dados de alta y permanecen asintomáticos y sin regresión tumoral a 5 años del primer caso y 18 del ultimo. La ecocardiografía transtorácica bidimensional continúa siendo un método diagnóstico de elección para estos casos.

Palabras clave: Tumores cardiacos, tratamiento quirúrgico, ecocardiografía transtorácica, pediatría.

\section{INTRODUCCION}

Los tumores cardiacos son infrecuentes en la edad pediátrica, antes de los avances de los métodos de diagnósticos imageneológicos la incidencia real era desconocida; con la ecocardiografía como herramienta diagnostica fundamental se aproxima al $0.08 \%$. $^{(1,2)}$ Nadas y Elllison en su revisión del Hospital de Niños de Boston reportan una incidencia del $0.027 \%$, en una serie de 11,000 autopsias $^{(3)}$. Los tumores cardiacos presentes en la infancia en su mayoría son primarios, benignos $(90 \%)$ y, además, poco sintomáticos, McAllister y Fenoglio refieren que los tumores cardíacos primarios se dividen en: $75 \%$ benignos y $25 \%$ malignos; más del $75 \%$ estos tumores benignos se encuentran en cavidades izquierdas ${ }^{(4)}$. Los tumores malignos predominantemente se encuentran ocupando las cavidades derechas al igual que los tumores secundarios o metastásicos que se encuentran en $60 \%$ ocupando las cavidades derechas. Los tumores secundarios o metastásicos representan menos del $10 \%$ de los casos ${ }^{(5,6)}$. Los tipos anatomopatológicos más frecuentes son: a) Rabdomiomas (45-75\%), generalmente se presentan como nódulos ventriculares únicos o múltiples, a veces intramurales b) Fibromas (6-25\%) por lo general son nódulos intramurales, únicos, más frecuentemente en ápex ventricular izquierdo, $\mathrm{y}$, pueden afectar a las paredes y/o las válvulas e incluso a las cavidades, aparecen en neonatos y lactantes. c) Mixomas (5-10\%). Pueden verse en niños mayores y adolescentes, más frecuentes en adultos; se presentan como masas únicas de la aurícula izquierda (75\%) o derecha (25\%), generalmente pedunculadas y frecuentemente calcificadas. d)Teratomas (2-10\%). La mayoría son intrapericárdicos y se caracterizan por ser masas únicas con múltiples quistes, unidas a la base del asymptomatic and without tumor regression at 5 years, in the first case and 18 in the last. Two-dimensional transthoracic echocardiography continues to be the diagnostic method of choice for these cases.

Keywords: Cardiac tumors, surgical treatment, transthoracic echocardiography, pediatrics.

corazón, más frecuentemente a los grandes vasos, donde a veces producen obstrucción, e) Hemangiomas: infrecuentes masas nodulares subendocárdicas; generalmente situadas en el septo interventricular y la aurícula derecha. Pueden asociarse a hemangiomas en otras localizaciones. $\mathrm{f}$ ) Tumor de células de Purkinje: de escasa frecuencia, estos tumores son masas Hamartomatosas de pequeño tamaño localizadas en el sistema de conducción y en el miocardio del ventrículo izquierdo. g) Sarcomas. Son los tumores primarios malignos más habituales. Proceden de estructuras musculares, fibrosas o vasculares, el más frecuente es el Angiosarcoma ${ }^{(7,8)}$.

La presentación clínica varía según la edad, del tipo de tumor y localización intracardiaca y estructuras que involucren, pudiendo algunos evolucionar desfavorablemente por los efectos hemodinámicos o mecánicos compresivos que pudieran presentar; de esta manera los pacientes pueden estar completamente asintomáticos o debutar con un evento de muerte súbita. Además pueden ser la manifestación de un trastorno genético como la esclerosis tuberosa ${ }^{(9,10)}$.

Debido a la gran variedad de alteraciones fisiopatológicas que pueden causar los tumores cardiacos el diagnóstico puede ser un hallazgo casual en la realización del ecocardiograma o por la presencia de trastornos del ritmo, fenómenos tromboembólicos o soplo cardiaco. El Electrocardiograma es inespecífico al igual que la Radiografía de Tórax.

La Ecocardiografía convencional (Modo M, Bidimensional, Doppler Pulsátil, Continuo y Color), 
cuenta con nuevos e importantes avances de precisión morfológica en reconstrucciones TriTetradimensionales, nuevas formas de evaluaciones funcionales como ecocardiografía con contraste, speckle tracking, strain rate, Doppler Tisular, etc., la han convertido en el método de elección en el diagnostico ya que determina la localización, extensión, repercusión hemodinámica y características del tumor, y permiten continuar el seguimiento con o sin resección quirúrgica ${ }^{(11)}$. Sin embargo, cuando se necesiten otros métodos de diagnóstico por imagen no invasivos, como la Resonancia Magnética Cardiaca(RMC) o Angiotomografia cardiaca (TCC) pueden ser de un valor complementario. La RMC ofrece ventajas adicionales como mejor definición para diagnosticar masas y tumores cardiacos de acuerdo a la densidad de los tejidos involucrados, las reconstrucciones tridimensionales y cortes multiplanares pueden aportar informaciones útiles para abordajes quirúrgicos asi como terapias adicionales; presentan un campo de visión más amplia, con alta sensibilidad y precisión de definición de invasión a estructuras adyacentes; tiene además excelente resolución de contraste. En los pacientes en quienes se encuentren contraindicados la RMC se puede realizar la TCC, siendo ésta una alternativa de igual valor que la RMC, dado que puede aportar prácticamente los mismos datos adicionales ante la sospecha de tumores malignos o metastásicoses prácticamente un estudio obligatorio en sospecha de malignidad primaria o metastásica, hacer un barrido tomográfico para estratificar el grado de invasión del tumor. Se tiene como factor limitante la exposición a la radiación ${ }^{(12)}$.

Los tumores cardiacos pueden causar obstrucciones al flujo de entrada o de salida del ventrículo izquierdo o derecho, obstrucción venosa, embolia pulmonar o sistémica, arritmias, hemolisis y derrame pericárdico ${ }^{(13)}$. El pronóstico depende de la localización y el tamaño, más que con la anatomía patológica, en caso de ser binigno. La intervención quirúrgica está determinada por los efectos hemodinámicos que ocasiona. Recordar, que la mayoría de estos pacientes se asocian a esclerosis tuberosa por lo que requieren atención de un equipo multidisciplinario.

EL objetivo del presente estudio es reportar una serie de casos de Tumores cardiacos que fueron sometidos a tratamiento quirúrgico, formas de presentación, diagnóstico y resultados

\section{MATERIALES Y MÉTODOS}

Análisis retrospectivo, observacional y transversal mediante la revisión de datos de historias clínicas de niños diagnosticados por ecocardiografía bidimensional transtorácico en el Servicio de Cardiología del Hospital General Pediátrico Niños de Acosta $\tilde{N u}$, dependiente del Ministerio de Salud Pública; en un periodo de 5 años.

Los diagnósticos ecocardiográficos fueron todos transtorácicos con equipo Philips X Matrix IE33, con transductores S8-3 y X5-1. Se realizo barrido tomográfico en un solo pacte con sospecha clínica de malignidad.

El tipo de tumor fue identificado por anatomía patológica.

Descripción de los Casos Clínicos: Tabla 1. Caso 1: Niña preadolescente de 12 años, que consulta por episodios frecuentes de chillido de pecho e infecciones respiratorias, con poca mejoría al tratamiento sintomático; además disnea de esfuerzo a moderados esfuerzos, astenia, anorexia, fiebre intermitente y pérdida de peso importante en los últimos 2 meses. A la auscultación cardiovascular presentó soplo sisto-diastólico en foco mitral IV/VI; por lo que se solicitó una ecocardiografía cuyo hallazgo más significativo fue la constatación de una enorme masa tumoral que asienta en aurícula izquierda de 50 × 60 milímetros de diámetro y que obstruía de forma dinámica la vía de entrada del ventrículo izquierdo, con disfunción moderada del ventrículo con una fracción de eyección de $45 \%$, además de constatarse una hipertensión pulmonar de $80 \mathrm{mmHg}$. Al momento de la internación se encontraba en ortopnea e insuficiencia cardiaca con facies sufriente, cianosis peribucal y facies rubicunda, taquicárdica 190 latidos por minuto con tendencia a hipotensión, 77/50 mmHg. Pulmones con ausencia de murmullo vesicular en ambos campos pulmonares y hepatomegalia a 4 centímetros del reborde costal derecho. Fue sometida a cirugía cardiaca de urgencia, donde se constató el enorme 
tumor que cubría completamente la vía de entrada ocasionando obstrucción mecánica con gran dilatación de la aurícula izquierda $(70 \mathrm{~mm})$, no obstante, fue posible realizar la extracción total del tumor además de una reconstrucción y plastia de la valva Mitral. El postoperatorio fue muy dificultoso por persistencia de hipertensión pulmonar y disfunción biventricular transitoria que con la
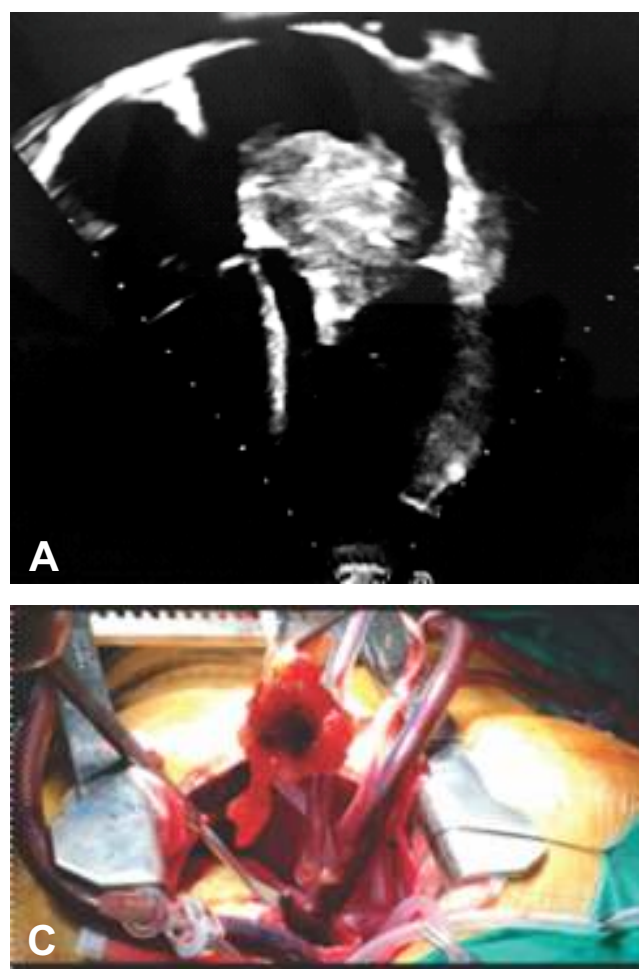

administración de Óxido nítrico inhalatoria y la asistencia de ECMO (asistencia circulatoria por membrana extracorpórea) por 13 días obtuvo una mejoría que posiblitó darla de a la cuarta semana del postoperatorio en buenas condiciones generales, a 20 meses poscirugía se encuentra en clase funcional I (NYHA). El estudio anatomopatológico informó tratarse de un Mixoma Auricular (Fig 1).
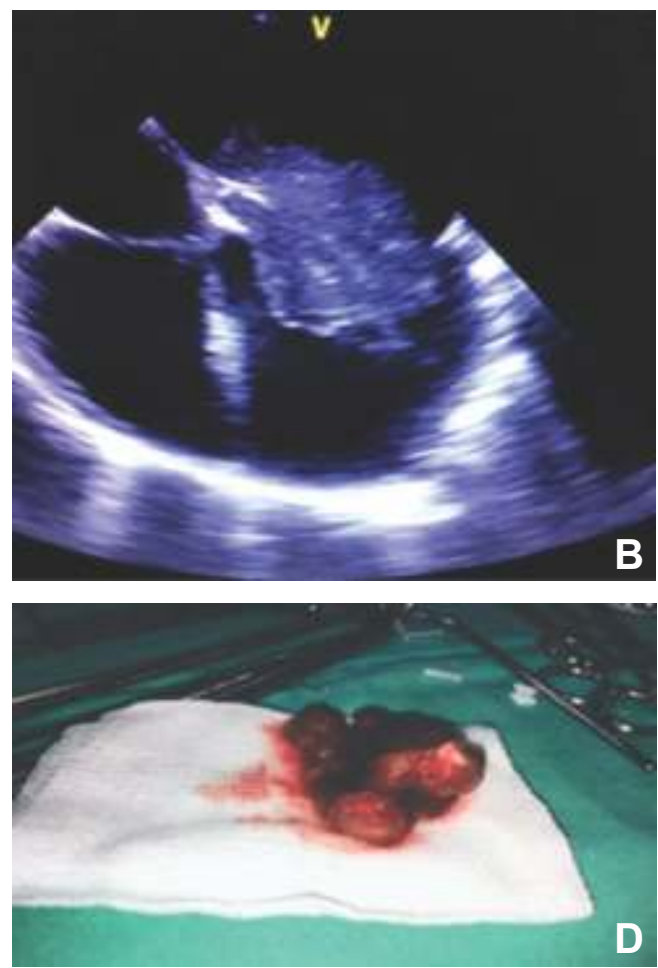

Figura 1. Caso 1 A) Ecocardiografía transtorácica bidimensional en cuatro cámaras que muestra masa tumoral en Aurícula izquierda, ocluyendo totalmente la vía de entrada al ventrículo izquierdo. B) Ecocardiografía transesofágica preoperatoria que muestra el tumor previa resección. C) Extracción de masa tumoral. D) Pieza extraída de masa tumoral.

Caso 2: Niña, lactante menor de 1 año 2 meses de edad, consulta por episodio de taquicardia persistente. Se realiza ecocardiografía bidimensional transtorácico que revela la presencia de una gran masa tumoral encapsulada de 50 milímetros aproximadamente que dilata la Aurícula derecha, protruye al ventrículo derecho ocasionando una insuficiencia tricúspide importante, se somete a cirugía con circulación extracorpórea y se extirpa el tumor en su totalidad. El informe del estudio histopatológico revela a la Macroscopia: nódulo bien delimitado, blanquecino grisáceo de $4.5 \mathrm{~cm}$ de diámetro mayor de consistencia solida elástica que al corte muestra banda fibrótica paracentral y en su alrededor parénquima pseudonodular. En la periferia se observa fina capsula fibrosa. Diagnóstico histológico: (Figura 2) Rabdomioma auricular sin cambios atípicos. La capsula referida en la macroscopía se halla conformada por tejido muscular cardiaco sin atipias, en el post- operatorio inmediato presentó Taquicardia supraventricular Paroxística que cede con medicación. Fue dada de alta al $5^{\circ}$ día post-operatorio en excelentes condiciones clínicas. (Fig 2) 

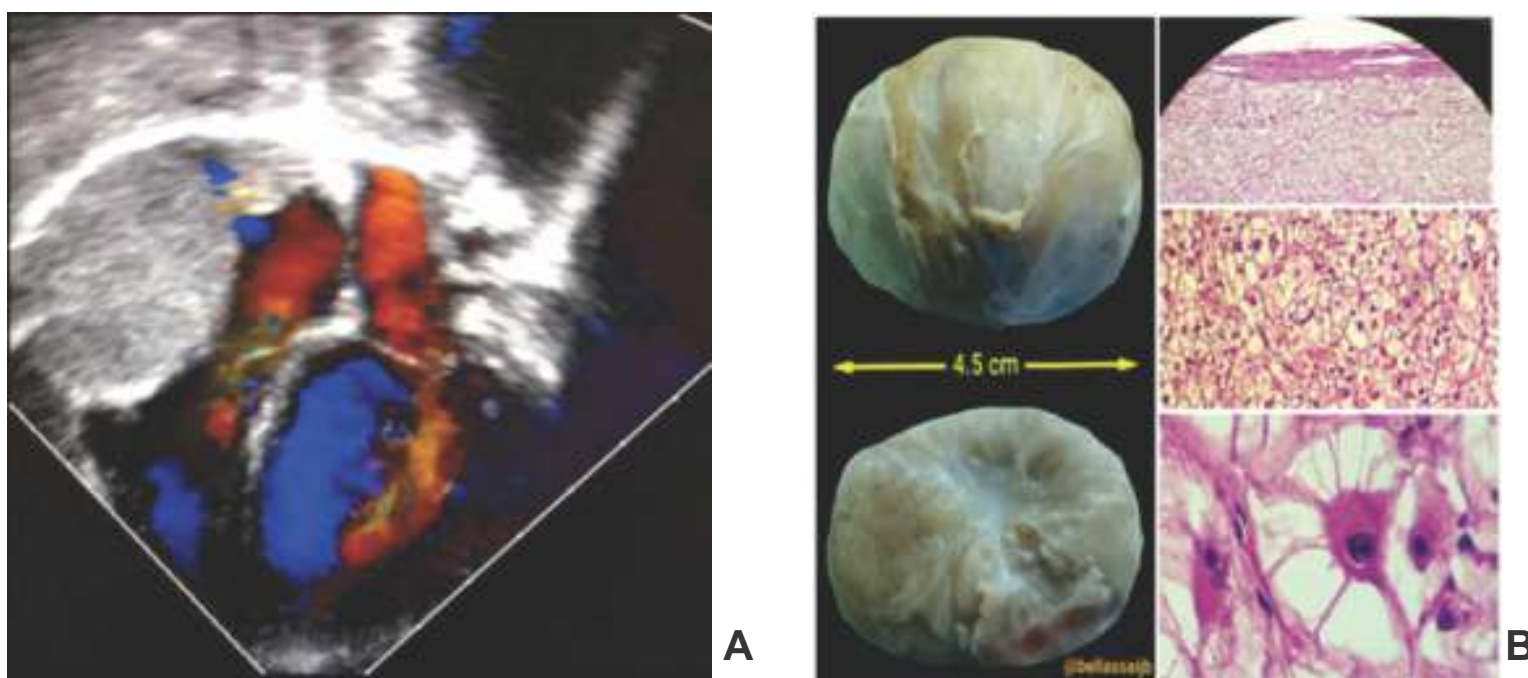

Figura 2. Caso 2 A) Ecocardiografía transtorácica bidimensional en 4 cámaras que muestra masa gigante que ocupa las 2/3 partes de la Aurícula Derecha e invade vía de entrada de Ventrículo derecho. B) Imagen de anatomía patológica que corresponde al tumor extirpado.

Caso 3: Varón preadolescente de 11 años, asintomático que se realizó un chequeo cardiovascular preoperatorio, y, cuyo hallazgo de Extrasístoles Ventriculares Bigeminadas frecuentes, motivó la realización de una Ecocardiografía con hallazgo de tumoraciones gigantes y numerosas en ventrículo izquierdo que asentaban dos de ellas en los músculos papilares del ventrículo y que hemodinámicamente protruían a nivel de la válvula mitral; fue intervenido con circulación extracorpórea, para la exéresis total de los tumores. Sin complicaciones en el periodo

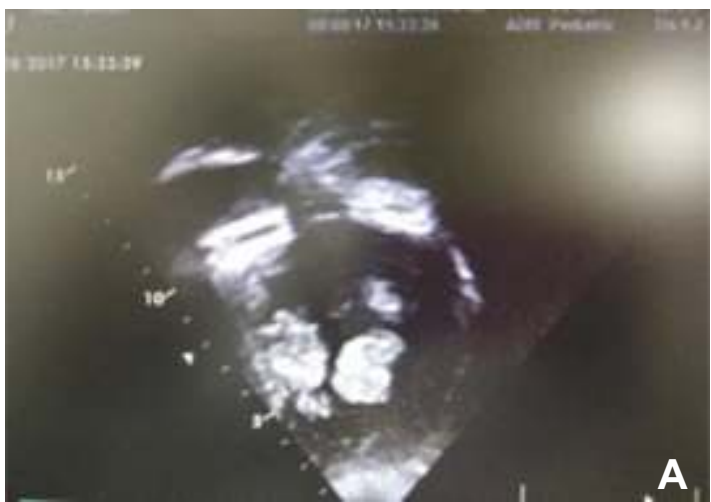

postoperatorio. La anatomía patológica informó Macroscopia: Nodulo blanquecino bien delimitado $2.5 \times 2 \times 1.5 \mathrm{~cm}$ de superficie extensa lisa y que al corte muestra parénquima blanquecino amarillento de aspecto mixoide Microscopia: nódulo bien delimitado conformado por tejido fibroso hialino bien vascularizado con áreas de células mixomatosas, sin signos de malignidad. Diagnóstico histopatológico: Mixoma Ventricular. Alta al sexto día de cirugía y a tres años del mismo permanece en clase funcional I (NYHA). (Fig 3)

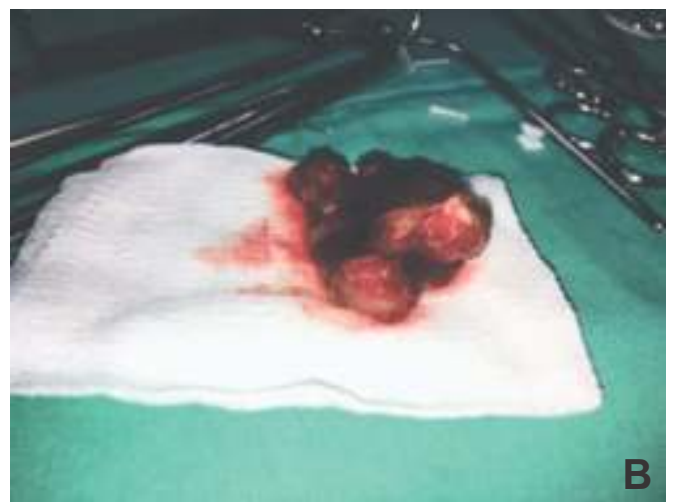

Figura 3. Caso 3. A) Ecocardiografía bidimensional: se visualizan múltiples tumores de diferentes tamaños localizados en el ventrículo izquierdo. B) pieza quirúrgica de uno de los tumores extraídos del ventrículo.

Caso 4: Niña preadolescente de 11 años de edad, remitida a consultorio de cardiología infantil por soplo cardiaco y cansancio a los grandes esfuerzos; al examen físico se ausculta soplo sistólico y diastólico "en arrullo de paloma" en foco mitral III/VI, $2^{\circ}$ ruido hiperfonético, pulsos periféricos 
palpables. La Ecocardiografía transtorácica evidenció una imagen hiperecogénica multilobulada de $55 \mathrm{~mm}$, que se inicia en el septo interauricular bajo y que asienta sobre la válvula mitral -hoja antero-septal- ocluyendo el orificio en forma dinámica en aproximadamente $50 \%$. Se somete a cirugía con circulación extracorpórea para exéresis tumoral, al hallazgo quirúrgico se demostró que la masa tumoral estaba completamente adherida a la vávlula mitral, por lo cual requirió el reemplazo de la válvula mitral por prótesis biológica. El estudio anatomopatológico informó tratarse de un Mixoma Auricular. Permaneció internada por 8 días en la unidad de cuidados intensivos cardiológicos, sin complicaciones.

Caso 5: niño escolar de 9 años que realiza chequeo cardiovascular para práctica deportiva, aparentemente asintomático, se realizan Electrocardiograma que fue normal y una Ecocardiografía con el hallazgo de derrame pericárdico posterior tabicado y una imagen quística tabicada gigante retrocardiaca con compromiso de pericardio y pleura de contenido liquido de $65 \mathrm{~mm} \mathrm{x}$ $35 \mathrm{~mm}$ de forma semilunar, en su interior se observaba una imagen de masa sólida, homogénea, sospechoso

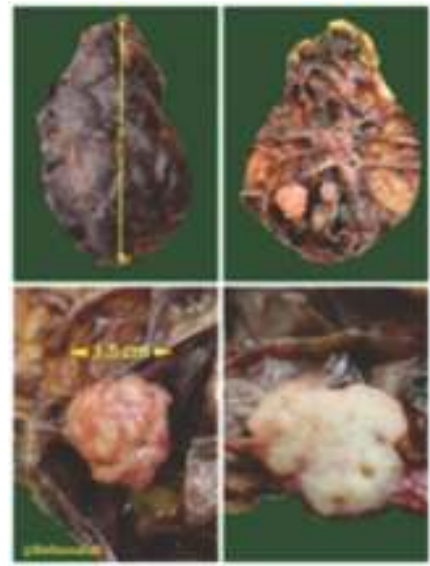

de ser una masa tumoral de aproximadamente $15 \mathrm{~mm}$. Se complementó con Tomografia Torácica con contraste para mayores precisiones técnicas, y que confirmaba los mismos hallazgos descritos por la ultranosografía cardiaca. Por toracotomía anterior se drena el derrame pericárdico multitabicado con importante cantidad de líquido turbio verdoso, en su interior se descubre un quiste de contenido líquido y, en el interior de este quiste una masa tumoral solida que se extirpa, midiendo $12 \times 6 \times 2 \mathrm{~cm}$ de superficie extensa beige, rojo vinosa que al corte deja escurrir liquido sanguinolento que una vez evacuado deja ver cavidad quística de pared milimétrica traslucida que hacia uno de los polos presenta formación nodular polipoide de $1.5 \mathrm{~cm}$ de diámetro mayor, blanquecina bien delimitada al corte esta formación se muestra compacta con algunos microquistes. El estudio anatomopatlógico arroja como diagnóstico: “Quiste tímico con carcinoma mucoepidermoide de Timo de bajo grado de malignidad intraquístico, resecado en su totalidad, sin infiltración parietal. Pared fibrosa sin cambios atípicos". Evoluciona sin complicaciones en el post-operatorio inmediato, siendo dado de alta al 6to día post-operatorio. A 18 meses de cirugía se encuentra asintomático, sin reincidencia del tumor. (Fig 4)

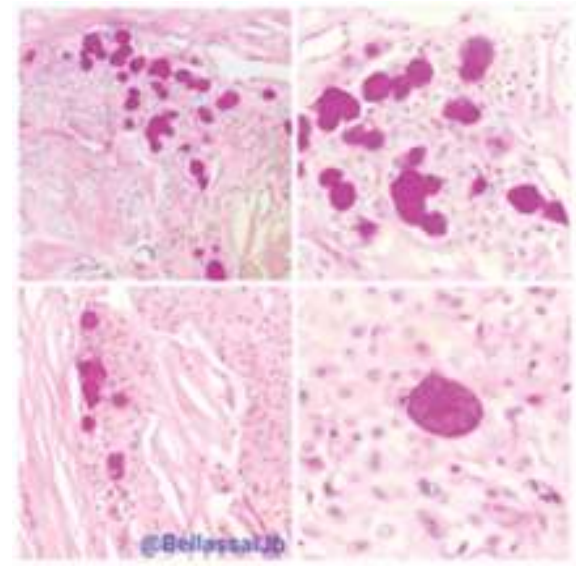

Figura 4. Caso 5: A) Pieza de anatomía patológica extraída en su totalidad alojado dentro del pericardio y que corresponde a un Tumor epidermoide de timo de bajo grado de malignidad. B) Estudio histopatológico del mismo tumor.

\section{RESULTADOS}

En nuestra casuística se incluyeron 5 pacientes la edad media de 8.8 años, relación F:M 3:2. En 3/5 pacientes fue por hallazgo casual y el restante y $2 / 5$ presentó síntomas relacionados con la ubicación anatómica del tumor. Hubo $100 \%$ de coincidencia del hallazgo por Ecocardiografía bidimensional transtorácico de imagen compatible con Tumor con el hallazgo quirúrgico el diagnóstico histopatológico Estos 
fueron; Mixoma en $3 / 5$ 1/5 Rabdomioma gigante y 1 /5 Carcionoma Mucoepidermoide de Timo.

El $80 \%$ de los tumores fue intracardiaco por lo que requirió cirugía cardiaca con circulación extracorpórea. En un caso se realizó sin circulación extracorpórea por que el tumor se encontraba adherido al pericardio. La evolución de los pacientes en el periodo posoperatorio inmediato fue satisfactoria con un promedio de alta de 6 días en $4 / 5$ pacientes y en un caso 25 días de internación por ICC IV/IV (NYHA) e Hipertensión Pulmonar previa a la cirugía, cursando con bajo gasto cardiaco -derechoe Hipertensión pulmonar severa en el postoperatorio inmediato. Estuvo en ECMO por 13 días.
No se registró mortalidad intra o postoperatoria y todos los pacientes que presentaron síntomas tuvieron mejorías clínicas notorias con mejoría de la clase funcional. En caso del niño que presento un Quiste Tímico con carcinoma mucoepidermoide de bajo grado, gracias a la detección precoz y la resección total del tumor no necesitó ningún tratamiento adicional. Este tumor es raro, en la literatura médica se registra unos 30 casos publicados $^{(14)}$.

Los datos demográficos, motivos de consulta y diagnostico histopatológico de los tumores se resumen en la Tabla 1.

Tabla 1. Tumores cardiacos primarios, en esta serie de casos.

\begin{tabular}{ccccccc}
\hline $\mathbf{N}^{\circ}$ DE CASOS & EDAD DE Dx & $\mathbf{S}$ & $\mathbf{P}$ & MC & LOCALIZACIÓN & TIPO \\
\hline 1 & $12^{\mathrm{a}}$ & $\mathrm{F}$ & $46 \mathrm{k}$ & ICC & Auricula izquierda & Mixoma \\
\hline 2 & $1 \mathrm{a} 2 \mathrm{~m}$ & $\mathrm{~F}$ & $10 \mathrm{k}$ & Arritmias & Aurícula derecha & $\begin{array}{c}\text { Rabdomioma } \\
\text { gigante }\end{array}$ \\
\hline 3 & $11^{\mathrm{a}}$ & $\mathrm{M}$ & $56 \mathrm{k}$ & $\begin{array}{c}\text { ECG } \\
\text { alterado }\end{array}$ & $\begin{array}{c}\text { Ventrículo } \\
\text { Izquierdo }\end{array}$ & $\begin{array}{c}\text { Mixomas } \\
\text { (multiples en racimo) }\end{array}$ \\
\hline 5 & $11^{\mathrm{a}}$ & $\mathrm{F}$ & $31 \mathrm{k}$ & $\begin{array}{c}\text { Soplo } \\
\text { cardiaco }\end{array}$ & Valvula Mitral & $\begin{array}{c}\text { Mixoma } \\
\text { gigante }\end{array}$ \\
\hline 5 & $9^{\text {a }}$ & $\mathrm{M}$ & $27 \mathrm{k}$ & $\begin{array}{c}\text { Evaluación } \\
\text { pre deportiva }\end{array}$ & Pericardio & $\begin{array}{c}\text { Carcinoma } \\
\text { mucoepidermoide }\end{array}$ \\
\hline
\end{tabular}

\section{DISCUSIÓN}

Se presentan los casos de tumores cardiacos, no frecuentes en pediatría, en concordancia con la literatura la mayoría fueron benignos ${ }^{(15)}$.

El tratamiento convencional es, en general el seguimiento de los mismos es decir tratamiento médico clínico; pero la intervención quirúrgica es necesaria cuando producen efectos hemodinámicos adversos, compresiones mecánicas, arritmias cardiacas secundarias a la presencia de los mismos en las diferentes estructuras cardiovasculares o disfunción ventricular, tal como los casos presentados $^{(16)}$. En algunos casos los tumores benignos cardiacos pueden producir adherencia o invasión de estructuras nobles como válvulas cardiacas, dificultando la extirpación del tumor en forma aislada. En esta situación se sacrifican estructuras cardiacas y se procede al reemplazo con prótesis tal como ocurrió en uno de los pacientes reportados en el presente estudio.

En caso de malignidad, la conducta a seguir necesariamente estará relacionada al origen del tumor primario y el estadio en que se encuentre. Uno de los casos reportados presento un tumor maligno de rara presentación. Fue diagnosticado de manera fortuita por un chequeo cardiovascular, que mostro y la presencia del derrame pericárdico, posiblemente secundaria a una reacción inflamatoria por la proximidad del proceso tumoral. El material fue extraído en su totalidad por lo cual no necesitó terapia adicional. En nuestra serie de casos el tumor más frecuente resulto ser el mixoma, y cabe mencionar que en ninguno de los casos se asoció esclerosis tuberosa.

La Ecocardiografía bi y tridimensional transtorácica 
en la edad pediátrica, y transesofágica en pacientes mayores o con mala ventana ultrasonográfica, continúa siendo el método gold standard para el

\section{REFERENCIAS BIBLIOGRAFICAS}

1. Driscoll D, Cetta F, Eidem BW. Tumores Cardiacos. En: Eidem WA, Cetta F, O'Leary P, editores. Ecocardiografía en Enfermedad Cardiaca Congénita Pediátrica y de Adultos. Venezuela: Amolca; 2014. p. 355-361.

2. Straus R, Merliss R. Prymari tumors of the hearts. Arch Pathol. 1945; 39:74-78.

3. Nadas AS, Ellison RC: Cardiac tumors in infancy. Am J Cardiol 1968; 21(3):363-366. DOI: https://www.doi.org/ 10.1016/0002-9149(68)90140-9

4. McAllister HA, Fenglio JJ. Tumor of the cardiovascular system. Atlas of tumor Pathology. Washington: Armed Force Institute of Pathology; 1978. p. 5-71.

5. Bjessmo S, Ivert T. Cardiac mixoma: 40 years experience in 63 patients. Ann Thorac Surgery. 1997; 63:697-700. DOI: https://www.doi.org/10.1016/s0003-4975(96)01368-9

6. Allaf D, Buretter R, Pierard L, Limet R. Cardiac taponade as the first manifestation of cardiothoracic malignancy a study of 10 case. Eur Heart J 1986; 7(3):247253. DOI: https://www.doi.org/10.1093/oxfordjournals. eurheartj.a062059

7. Primary pediatric cardiac tumors: A17 year experience. Cardiol Young. 1999; 9(2):155-162.

8. Patiño E. Tumores Cardiacos. En: Attie. F, Buendia A, Calderon J, Zabal C, editores. Cardiología Pediátrica. 2da ed. Mexico: Editorial Médica Panamericana; 2013. p.466-470.

9. Vazquez H, Quilindro A, Sicurello M, Bailatti N, Gómez J, Rodríguez A. Tumores cardíacos primarios en pediatría. Rev. Argent. Cardiol, 2003, 71(3):270-274.

10. Sabatine MS, Colucci WS, Shoen FJ. Tumores Cardíacos diagnóstico seguro y con suficientes precisiones técnicas ${ }^{(18)}$.

Primarios. En: Ziper DP, Lobby P, Bonew RO, Braunwald E, editores. Tratado de Cardiología. 7ma ed. Madrid; 2006. p. 1741-1755.

11. Sánchez Andrés A, Insa Albert B, Carrasco Moreno J, Cano Sánchez A, Moya Bonoza A, Sáez Palacios JM. Tumores cardíacos primarios en la infancia. An Pediatr (Barc) [internet]. 2008 [citado ene 2013]; 69(1):15-22.

12. Erdmenger Orellana J, Vázquez C, Ortega Maldonado J. Valor de la ecocardiografía en el diagnóstico contemporáneo de tumores cardíacos primarios en pediatría. Arch. Cardiol. Méx. 2005; 75(2):154-158.

13. Marx G, Moran A. Cardiac tumors. En: Huhg A, DriscollD, Shayddy R, Feltes T. Heart disease in infants, children and adolescent. $7 \mathrm{ma}$ ed. Baltimore: Williams and Wilkins; 2008.1425 p.

14. Patiño E, Calderon J, Buendia A, Juanico A. Giant aortic aneurysm and rhabdomyomas in infant whit tuberous sclerosis (case report). Arch Cardiol Mex 2005; 75:448

15. Nonaka D, Klimstra D, Rosai J. Thymic mucoepidermoid carcinomas: a clinicopathologic studybof 10 cases and review of the literature. The American Journal of Surgical Pathologhy. 2004; 28(11):1526-1531. DOI: https://www.doi.org/10.1097/01.pas.0000137999.23780.2a

16. Marantz P, Capella H, Micheli D, Berri G. Tumores cardíacos en la infancia. Diagnóstico y seguimiento por ecografía. Arch. argent. pediatr. 1992; 90:193-196.

17. Abad C. Tumores cardíacos (I): Consideraciones generales: Tumores primarios benignos. Rev Esp Cardiol. 1998; 51(1):10-20. 